\title{
Light-Matter Interaction and Hybrid Vector Breather
}

\author{
G. T. Adamashvili \\ Technical University of Georgia, \\ Kostava str.77, Tbilisi, 0179, Georgia. \\ email: guram_adamashvili@ymail.com.
}

\begin{abstract}
The nonlinear coherent interaction of light with the dispersive and Kerr-type third-order susceptibility medium containing optical impurity atoms or semiconductor quantum dots is considered. Using the generalized perturbation reduction method, the nonlinear wave equation is reduced to the coupled nonlinear Schrödinger equations. It is shown that the second-order derivatives play a key role in the description of the process of formation of the bound state of two breathers oscillating with the sum and the difference of frequencies and wave numbers. The resonant, nonresonant and hybrid mechanisms of the formation of the two-component nonlinear pulse - the vector breather are realized depending on the light and medium parameters. Explicit analytical expressions for the profile and parameters of the nonlinear pulse are presented. The conditions of the excitation of resonant, nonresonant and hybrid nonlinear waves are discussed. In the particular case, the resonant vector breather coincides with the vector $0 \pi$ pulse of self-induced transparency.
\end{abstract}

PACS numbers: 42.65.-k, 05.45.Yv, 02.30.Jr, 52.35.Mw

\section{INTRODUCTION}

The nonlinear light-matter interaction is a field of intensive research that covers a wide class of nonlinear optical phenomena. At the nonlinear coherent interaction of light and matter there occur various nonlinear processes. A special interest is shown in the processes which lead to the formation of nonlinear solitary waves of stable profile. The propagation of these waves is one of the most striking demonstrations of nonlinearity in optical media. The analysis of the mechanisms causing the formation of nonlinear solitary optical waves, the establishment of their types and the study of their properties in various nonlinear media are among the basic problems of the physics of nonlinear optical waves.

Depending on the character of nonlinearity, the nonresonant and resonant mechanisms of the formation of nonlinear solitary waves are considered. In the case of nonresonant nonlinearity, which can be connected with the quadratic (second-order) or cubic (third-order) nonlinear susceptibilities, its competition with dispersion or diffraction leads to the formation of nonresonant optical nonlinear waves of stable profiles which can be respectively classified as temporal or spatial solitary waves [1 8$]$. Resonant optical solitary waves can be formed in the conditions of selfinduced transparency (SIT) $9-19$.

Both resonant and nonresonant nonlinear solitary waves are divided into two basic types: single-component and two-component nonlinear waves. In the main, to single-component nonlinear solitary waves are attributed scalar solitons and scalar breathers, and to two-component waves there belong vector solitons and vector breathers or their modifications.

Although the SIT phenomenon and resonant nonlinear waves, namely, scalar solitons and scalar breathers have already been studied for quite a long time, in recent years the interest in their investigation has noticeably revived. A lot of theoretical and experimental studies have been carried out 20 44].

In SIT theory, second-order derivatives in space coordinate and the time of the electric field strength of the pulse in the Maxwell wave equation were usually neglected. So, it was believed that the basic SIT waves are the scalar single-component $0 \pi$ pulse and the scalar singe-component McCall-Hahn's $2 \pi$ pulse 9 -15]. However, the situation has recently changed due to the development of a new mathematical approach - the generalized perturbative reduction method (PRM) [45 56].

As different from the standard PRM [57] adapted for the consideration of single-component nonlinear waves, the generalized PRM makes it possible to proceed to the next stage of the development of SIT theory, i.e. to the study of the properties of two-component solitary waves. The generalized PRM demonstrates what an important role the second-order derivatives of the Maxwell wave equation play in SIT theory. These derivatives are used, in particular, to describe the interaction between two single-component breathers, which leads to the formation of their bound state. Using the generalized PRM, qualitatively new results were obtained in SIT theory. It was established that there exists a two-component vector breather (TVB) which in SIT theory is called the vector $0 \pi$ pulse. The vector $0 \pi$ pulse is the bound state of two breathers of the same polarization, one component oscillating with the sum and the other with the difference of frequencies and wave numbers (SDFW). As a result of such a superposition there arises a nonlinear zero-area pulse with specific phase modulation and the profile which significantly differs from the profiles of the scalar single-component SIT-soliton and the scalar single-component SIT-breather. This clearly implies that the basic SIT 
pulse is the vector $0 \pi$ pulse and the scalar $2 \pi$ pulse. But the scalar $0 \pi$ pulse of SIT is only a certain approximation of the vector $0 \pi$ pulse, which takes place only if the second-order derivatives in SIT equations are neglected or a less general mathematical approach than the generalized PRM, for instance, the standard PRM is used (for details see $[51-53])$.

The optical resonant vector $0 \pi$ pulse in various isotropic and anisotropic materials was investigated for one-photon and two-photon resonance excitations, plane and surface waves, also the waveguide modes were studied in different physical situations [45 50]. Optical nonresonant TVB oscillating with the SDFW in the dispersive and Kerr-type third-order susceptibility medium were considered using the generalized PRM [58].

Depending on the numerical values of light and medium parameters there may occur physical situations in which both resonant and nonresonant mechanisms of the formation of nonlinear waves act simultaneously. In that case, the hybrid (blended) mechanism of excitation of a nonlinear solitary wave becomes active and a hybrid nonlinear pulse can be formed. Hybrid single-component scalar solitons and hybrid single-component scalar breathers were investigated by different mathematical approaches in the course of many years [59 68]. However, using the generalized PRM we have obtained the novel result - the hybrid TVB oscillating with the SDFW which has not been studied until now.

The goals of the present study are as follows: investigation of processes of the formation of the optical hybrid TVB oscillating with the SDFW and propagating in dispersive Kerr-type nonlinear medium containing a small concentration of optical impurity atoms or semiconductor quantum dots (SQDs); derivation of explicit analytical expressions for the parameters of the hybrid TVB oscillating with the SDFW in the carrier wave frequency and wave number region; definition of the conditions for the existence of the nonlinear waves.

The rest of this paper is organized as follows. Section II is devoted to the derivation of SIT equations in dispersive Kerr-type medium containing optically active impurity atoms (SQDs) for slowly varying envelope functions. In Section III, using the generalized PRM the nonlinear wave equation for these functions is transformed to coupled nonlinear Schrödinger equations (NSEs) for auxiliary functions. The explicit expressions are obtained for the TVB oscillating with the SDFW. In the last section IV, we discuss the obtained results, the role of second-order derivatives in the process of formation of the bound state of two wave packets and consider the criteria of the existence of resonant, nonresonant and hybrid nonlinear waves.

\section{BASIC EQUATIONS}

We study the physical process of formation of the optical TVB oscillating with the SDFW in the dispersive Kerrtype third-order susceptibility medium containing a small concentration $n_{0}$ of optical active impurity atoms or SQDs. The optical linear-polarized along the $x$ axis pulse with width $T<<T_{1,2}$, frequency $\omega>>T^{-1}$ and wave vector $\vec{k}$ spreads in the positive direction along the z-axis. Here $\omega$ is the carrier wave frequency of the optical wave, while $T_{1}$ and $T_{2}$ are respectively the longitudinal and the transverse relaxation time of optical resonant atoms or SQDs. In general, the dispersion can be described by means of the electric permittivity tensor $\varepsilon_{i j}(z, t)$ which depends on the space coordinate $z$ and the time $t$. But in an isotropic medium the permittivity tensor reduces to the form $\varepsilon_{i j}(z, t)=\varepsilon(z, t) \delta_{i j}$, where $\varepsilon(z, t)$ is the scalar function, $\delta_{i j}$ is the Kronecker symbol.

The wave equation for the $x$-component of the strength of the electric field $\vec{E}(E, 0,0)$ is written in the form [5, 69]

$$
\mathfrak{c}^{2} \frac{\partial^{2} E}{\partial z^{2}}-\frac{\partial^{2} D_{l}}{\partial t^{2}}=4 \pi \frac{\partial^{2} P}{\partial t^{2}}
$$

where $\mathfrak{c}$ is the velocity of light in vacuum, $D_{l}$ is the linear part of the $x$-component of the electric displacement vector (Appendix I). The nonlinear polarization of the medium $P=P_{n}+P_{r}$, contains the nonresonant and resonant parts. Here

$$
\begin{gathered}
P_{n}=\int \rho_{x x x x}\left(z_{1}, z_{2}, z_{3}, t_{1}, t_{2}, t_{3}\right) E\left(z-z_{1}, t-t_{1}\right) E\left(z-z_{1}-z_{2}, t-t_{1}-t_{2}\right) \times \\
E\left(z-z_{1}-z_{2}-z_{3}, t-t_{1}-t_{2}-t_{3}\right) d z_{1} d z_{2} d z_{3} d t_{1} d t_{2} d t_{3}
\end{gathered}
$$

is the $x$-component of the nonresonant nonlinear Kerr-type polarization of the medium, $\rho_{x x x x}$ is the component of the third-order susceptibility tensor. $P_{r}=n_{0} \mu s_{1}$ is the $x$-component of the polarization of two-level optical impurity atoms (SQDs), where $\mu$ is the dipole matrix element of the optical atoms (SQDs), $s_{i}(t)=<\hat{\sigma}_{i}(t)>$ are average quantities of the Pauly's operators $\hat{\sigma}_{i}, \quad(i=1,2,3)$.

The dependence of the function $P_{r}$ on the strength of the electrical field $E$ is defined by the optical Bloch equations [11, 25, 70]

$$
\frac{\partial s_{1}}{\partial t}=-\omega_{0} s_{2}
$$




$$
\begin{gathered}
\frac{\partial s_{2}}{\partial t}=\omega_{0} s_{1}+\kappa_{0} E s_{3}, \\
\frac{\partial s_{3}}{\partial t}=-\kappa_{0} E s_{2}
\end{gathered}
$$

where $\kappa_{0}=\frac{2 \mu}{\hbar}, \hbar$ is Planck's constant, $\omega_{0}$ is the excitation frequency of optical impurity two-level atoms.

The system of equations (1)-(3), which are SIT equations in the dispersive Kerr-type medium, can be simplified by using the method of slowly varying profile. To this end, we write the $x$-component of the strength of the electric field $E$ and the polarization $P_{r}$ in the form [10 15$]$

$$
E=\sum_{l= \pm 1} \hat{E}_{l} Z_{l}, \quad P_{r}=n_{0} \mu \sum_{l= \pm 1} d_{-l} Z_{l}
$$

where $\hat{E}_{l}$ and $d_{l}$ are the slowly varying complex amplitudes of the optical electric field and the polarization of optical active atoms. These are complex functions in view of the fact that the wave is phase modulated. $Z_{l}=e^{i l(k z-\omega t)}$ is the fast oscillating function. Because $E$ is a real function, we set $\hat{E}_{l}=\hat{E}_{-l}^{*}$.

As compared with the carrier wave parts, the envelopes $\hat{E}_{l}$ and $d_{l}$ vary with a sufficient slowness in space and time. Therefore the following inequalities are valid for $\hat{E}_{l}$

$$
\left|\frac{\partial \hat{E}_{l}}{\partial t}\right| \ll \omega\left|\hat{E}_{l}\right|, \quad\left|\frac{\partial \hat{E}_{l}}{\partial z}\right| \ll k\left|\hat{E}_{l}\right|
$$

and similar inequalities hold for the complex function $d_{l}$.

Substituting Eq.(4) into the wave equation (1), and taking into account Eq.(17) (see Appendix I), we obtain the dispersion law for the propagating pulse in the medium

$$
\mathfrak{c}^{2} k^{2}=\omega^{2} \kappa
$$

and the nonlinear wave equation for the envelope function $\hat{E}_{l}$ in the form:

$$
\sum_{l= \pm 1} Z_{l}\left\{\left[i g_{1} \frac{\partial \hat{E}_{l}}{\partial z}+i g_{3} \frac{\partial \hat{E}_{l}}{\partial t}+g_{2} \frac{\partial^{2} \hat{E}_{l}}{\partial z^{2}}-g_{5} \frac{\partial^{2} \hat{E}_{l}}{\partial t^{2}}-g_{4} \frac{\partial^{2} \hat{E}_{l}}{\partial z \partial t}\right]-4 \pi \omega^{2} \sum_{l^{\prime}} \sum_{l^{\prime \prime}} \tilde{\rho}_{l, l^{\prime}, l^{\prime \prime}} \hat{E}_{l-l^{\prime}-l^{\prime \prime}} \hat{E}_{l^{\prime}} \hat{E}_{l^{\prime \prime}}\right\}+4 \pi \frac{\partial^{2} P_{r}}{\partial t^{2}}=0
$$

where

$$
\begin{gathered}
g_{1}=\omega^{2} a-2 l k \mathfrak{c}^{2}, \quad g_{2}=\omega^{2} c-\mathfrak{c}^{2}, \\
g_{4}=2 l \omega a+\omega^{2} \tilde{t}, \\
g_{5}=-\left(\omega^{2} d+2 l \omega b+\kappa\right) \\
\tilde{\rho}_{l, l^{\prime}, l^{\prime \prime}}=\int \rho_{x x x x}\left(z_{1}, z_{2}, z_{3}, t_{1}, t_{2}, t_{3}\right) e^{-i l\left(k z_{1}-\omega t_{1}\right)} e^{-i\left(l^{\prime}+l^{\prime \prime}\right)\left[k z_{2}-\omega t_{2}\right]} e^{-i l^{\prime \prime}\left[k z_{3}-\omega t_{3}\right]} d z_{1} d z_{2} d z_{3} d t_{1} d t_{2} d t_{3} .
\end{gathered}
$$

Eq.(7) describes various nonlinear processes which arise for the nonlinear coherent interaction of light and the dispersive Kerr-type medium containing optical impurity atoms (SQDs), and in particular, processes of the formation of single-component and two-component solitary nonlinear waves when resonant and nonresonant mechanisms act simultaneously.

\section{APPLICATION OF THE GENERALIZED PERTURBATION REDUCTION METHOD}

For the analyze of the two-component nonlinear solitary wave solution of Eq.(7) we apply the generalized PRM [45 51] by means of which this equation can be transformed to the coupled NSEs. In this approach the envelope function $\hat{E}_{l}(z, t)$ can be represented as:

$$
\hat{E}_{l}(z, t)=\sum_{\alpha=1} \varepsilon^{\alpha} \hat{E}_{l}^{(\alpha)}=\sum_{\alpha=1}^{\infty} \sum_{n=-\infty}^{+\infty} \varepsilon^{\alpha} Y_{l, n} f_{l, n}^{(\alpha)}(\zeta, \tau)
$$


where

$$
Y_{l, n}=e^{i n\left(Q_{l, n} z-\Omega_{l, n} t\right)}, \quad \zeta_{l, n}=\varepsilon Q_{l, n}\left(z-v_{g ; l, n} t\right), \quad \tau=\varepsilon^{2} t, \quad v_{g ; l, n}=\frac{d \Omega_{l, n}}{d Q_{l, n}}
$$

$\varepsilon$ is a small parameter. Such an expansion allows us to separate from $\hat{E}_{l}$ the even more slowly changing auxiliary function $f_{l, n}^{(\alpha)}$. It is assumed that the quantities $\Omega_{l, n}, Q_{l, n}$, and $f_{l, n}^{(\alpha)}$ satisfy the inequalities:

$$
\begin{gathered}
\omega \gg \Omega_{l, n}, \quad k \gg Q_{l, n}, \\
\left|\frac{\partial f_{l, n}^{(\alpha)}}{\partial t}\right| \ll \Omega_{l, n}\left|f_{l, n}^{(\alpha)}\right|, \quad\left|\frac{\partial f_{l, n}^{(\alpha)}}{\partial z}\right| \ll Q_{l, n}\left|f_{l, n}^{(\alpha)}\right| .
\end{gathered}
$$

for any value of indexes $l$ and $n$.

Although the quantities $Q_{l, n}, \Omega_{l, n}, \zeta_{l, n}$ and $v_{g ; l, n}$ depend on $l$ and $n$, for simplicity, we omit these indexes in the equations below when this does not cause confusion.

The generalized PRM (8) holds for the phase modulated complex function $\hat{E}_{l}$. Otherwise, if the wave is not phase-modulated, then the function $\hat{E}_{l}=\hat{E}_{-l}=\hat{E}$ is real and does not depend on the index $l$.

It should be noted that the generalized PRM is a sufficiently general mathematical approach and can be used not only for studying two-component solitary optical waves, but also for the investigation of two-component waves in nonlinear acoustics, hydro-dynamics, plasma physics, and so on [54 56, 71 73].

Substituting Eq.(8) into Eq.(7), and taking into account the explicit form of the polarization envelope (18), we obtain

$$
\begin{gathered}
\sum_{l= \pm 1} \sum_{\alpha=1}^{\infty} \sum_{n=-\infty}^{+\infty} \varepsilon^{\alpha} Y_{l, n} Z_{l}\left\{\tilde{W}_{l, n}+i \varepsilon J_{l, n} \frac{\partial}{\partial \zeta}+i \varepsilon^{2} h_{l, n} \frac{\partial}{\partial \tau}+\varepsilon^{2} H_{l, n} \frac{\partial^{2}}{\partial \zeta^{2}}\right\} f_{l, n}^{(\alpha)} \\
=\varepsilon^{3} \sum_{l= \pm 1} Z_{l} R_{l}\left[\left(\left|f_{l, l}^{(1)}\right|^{2}+2\left|f_{l,-l}^{(1)}\right|^{2}\right) f_{l, l}^{(1)} Y_{l, l}+\left(\left|f_{l,-l}^{(1)}\right|^{2}+2\left|f_{l, l}^{(1)}\right|^{2}\right) f_{l,-l}^{(1)} Y_{l,-l}\right] \\
\left.-\varepsilon^{3} i æ_{2} \sum_{l= \pm 1} l Z_{l} \int \frac{\partial \Theta_{l}^{(1)}}{\partial t} \Theta_{-l}^{(1)} \Theta_{l}^{(1)} d t^{\prime}+\ldots\right\}
\end{gathered}
$$

where

$$
\begin{gathered}
\tilde{W}_{l, n}=g_{3} n \Omega-g_{1} n Q-g_{2} Q^{2}+g_{5} \Omega^{2}-g_{4} Q \Omega+æ_{1} \frac{l n}{\Omega}, \\
J_{l, n}=g_{3} v_{g}-g_{1}-2 g_{2} n Q+2 n g_{5} \Omega v_{g}-g_{4} n\left(Q v_{g}+\Omega\right), \\
h_{l, n}=g_{3}+2 g_{5} n \Omega-g_{4} n Q \\
H_{l, n}=Q^{2}\left(g_{2}-g_{5} v_{g}^{2}+g_{4} v_{g}\right), \\
æ_{1}=\frac{4 \pi \omega^{2} n_{0} \mu^{2}}{\hbar}<g> \\
æ_{2}=\frac{8 \pi n_{0} \mu^{4}}{\hbar^{3}}<g> \\
R_{l}=4 \pi \omega^{2}\left(\tilde{\rho}_{l, l, l}+\tilde{\rho}_{l, l,-l}+\tilde{\rho}_{l,-l, l}\right) .
\end{gathered}
$$


To define the function $f_{l, n}^{(\alpha)}$ in Eq.(9), we equate to zero the terms corresponding to the same powers of $\varepsilon$. As a result, we obtain a sequence of equations. Starting with first order of $\varepsilon$, we find that only the components $f_{ \pm 1, \pm 1}^{(1)}$ or $f_{ \pm 1, \mp 1}^{(1)}$ of the function $f_{l, n}^{(1)}$ are different from zero. The relations between the parameters $\Omega_{l, n}$ and $Q_{l, n}$ is defined by Eq.(9) and has the form

$$
g_{3} n \Omega_{l, n}-g_{1} n Q_{l, n}+æ_{1} \frac{l n}{\Omega_{l, n}}=g_{2} Q_{l, n}^{2}-g_{5} \Omega_{l, n}^{2}+g_{4} Q_{l, n} \Omega_{l, n} .
$$

Substituting Eq.(11) into Eq.(9), we prove that the following equations hold $J_{ \pm 1, \pm 1}=J_{ \pm 1, \mp 1}=0$. From Eq.(9), to third order in $\varepsilon$, and introducing the functions $u_{ \pm 1}=\varepsilon f_{+1, \pm 1}$, we get the coupled NSEs in the form

$$
\left[i\left(\frac{\partial u_{ \pm 1}}{\partial t}+v_{ \pm} \frac{\partial u_{ \pm 1}}{\partial z}\right)+p_{ \pm} \frac{\partial^{2} u_{ \pm 1}}{\partial z^{2}}+\mathfrak{q}_{ \pm}\left|u_{ \pm 1}\right|^{2} u_{ \pm 1}+r_{ \pm}\left|u_{\mp 1}\right|^{2} u_{ \pm 1}=0\right.
$$

where

$$
\begin{gathered}
v_{ \pm}=v_{g ;_{+1, \pm 1}}, \quad p_{ \pm}=\frac{H_{+1, \pm 1}}{h_{+1, \pm 1} Q_{ \pm}^{2}}, \quad \mathfrak{q}_{ \pm}=\frac{\kappa_{1}^{ \pm}}{h_{+1, \pm 1}}, \quad r_{ \pm}=\frac{\kappa_{2}^{ \pm}}{h_{+1, \pm 1}} \\
\kappa_{1}^{ \pm}=R_{+1} \pm \frac{æ_{2}}{\Omega_{ \pm}^{3}}, \\
\kappa_{2}^{ \pm}=2 R_{+1} \pm \frac{æ_{2}}{\Omega_{ \pm}^{2} \Omega_{\mp}}\left(\frac{\Omega_{ \pm}}{\Omega_{\mp}}-1\right) .
\end{gathered}
$$

From Eq. (11) it follows that

$$
\begin{aligned}
& \Omega_{+1,-1}=\Omega_{-1,+1}=\Omega_{-}, \quad \Omega_{+1,+1}=\Omega_{-1,-1}=\Omega_{+}, \\
& Q_{+1,-1}=Q_{-1,+1}=Q_{-}, \quad Q_{+1,+1}=Q_{-1,-1}=Q_{+} .
\end{aligned}
$$

Substituting the components of the vector soliton $u_{+1}$ and $u_{-1}$ from Eq.(19) into Eqs.(4) and (8), for the $x$ component of the electric field $E(z, t)$ we obtain the TVB oscillating with the SDFW in the form

$$
E(z, t)=\frac{2}{\mathfrak{b} T} \operatorname{sech}\left(\frac{t-\frac{z}{V}}{T}\right)\left\{f_{+1} \cos \left[\left(k+Q_{+}+k_{+1}\right) z-\left(\omega+\Omega_{+}+\omega_{+1}\right) t\right]+f_{-1} \cos \left[\left(k-Q_{-}+k_{-1}\right) z-\left(\omega-\Omega_{-}+\omega_{-1}\right) t\right]\right\}
$$

\section{CONCLUSIONS AND DISCUSSIONS}

We consider the coherent nonlinear interaction of light with the dispersive Kerr-type medium containing optical impurity atoms or SQDs. It is shown that along with one-component nonlinear waves (solitons and breathers) investigated earlier [59 68], the two-component nonlinear wave (vector breather) Eq. (15) may also be formed when both resonant and nonresonant mechanisms of the formation of the nonlinear waves act simultaneously.

Eq.(15) is the TVB oscillating with the SDFW which is the solution of the nonlinear wave equation (11). This expression can be considered as a superposition of two small amplitude breathers. The first term of Eq.(15) is the small amplitude $\left(f_{+1}\right)$ breather oscillating with the sum of frequencies $\omega+\Omega_{+}$and wave numbers $k+Q_{+}$, and the second term is the small amplitude $\left(f_{-1}\right)$ breather oscillating with the difference of frequencies $\omega-\Omega$ numbers $k-Q_{-}$(taking into account Eq.(21)). The nonlinear coupling between these two wave packets is defined by the cross terms $r_{+}\left|u_{-1}\right|^{2} u_{+1}$ and $r_{-}\left|u_{+1}\right|^{2} u_{-1}$ of Eq.(12). Both breathers are polarized along the $x$ axis. The parameters of the breathers are closely interconnected with each other and are defined by Eqs.(13), (20) and (22). These breathers form the single integrated pulse Eq.(15) which propagates in a medium with the velocity $V_{0}$ (Appendix II). The dispersion relation and connections between the oscillating parameters $\Omega_{ \pm}$and $Q_{ \pm}$are defined by Eqs.(6), (11) and (14). Similar to the scalar single-component soliton and breather, the TVB oscillating with the SDFW Eq.(15) loses no energy while propagating through the medium.

In view of the fact that in different systems the quantity $æ_{2}$ in Eqs.(10) and (13) varies in a very wide range, we can consider in particular three different physical situations [59 68].

i. In the medium where the condition $R_{+1}<<\frac{\aleph_{2}}{\Omega_{ \pm}^{3}}$ is satisfied, the resonant TVB oscillating with the SDFW can be formed under the SIT condition. In that case, the TVB with the SDFW coincides with the vector $0 \pi$ pulse $[45-53$. 
ii. The nonresonant TVB oscillating with the SDFW can be formed when the light and medium parameters satisfy the condition $R_{+1}>>\frac{\mathfrak{x}_{2}}{\Omega_{ \pm}^{3}}[58]$.

iii. The hybrid TVB oscillating with the SDFW can be formed in a situation where the light and medium parameters satisfy the requirement that the quantities $R_{+1}$ and $\frac{\mathfrak{x}_{2}}{\Omega_{ \pm}^{3}}$ be of the same order.

Comparing Eqs.(15) and (7) we clearly see that the second-order derivatives $g_{2} \frac{\partial^{2} \hat{E}_{l}}{\partial z^{2}}-g_{5} \frac{\partial^{2} \hat{E}_{l}}{\partial t^{2}}-g_{4} \frac{\partial^{2} \hat{E}_{l}}{\partial z \partial t}$ are smaller than the first-order derivatives $i\left(g_{1} \frac{\partial \hat{E}_{l}}{\partial z}+g_{3} \frac{\partial \hat{E}_{l}}{\partial t}\right)$. Nevertheless the second-order derivatives play a significant role in the process of formation of nonlinear solitary waves. The second-order derivatives of Eq.(7) are connected with the terms $g_{2} Q_{l, n}^{2}-g_{5} \Omega_{l, n}^{2}+g_{4} Q_{l, n} \Omega_{l, n}$ of Eq.(11) and the characteristic parameters $\Omega_{ \pm}$and $Q_{ \pm}$of Eq.(14).

In the particular case, if we neglect the second-order derivatives in Eq.(17), as was usually done before the development of the generalized PRM, then we can obtain scalar single-component SIT-soliton and scalar single-component SIT-breather solutions of Eq.(77). Indeed, in this case, neglecting in Eq.(11) the terms with the coefficients $g_{2}, g_{4}$ and $g_{5}$, we see that this equation does not any longer depend on the indexes $l$ and $n$. Consequently, the conditions $\Omega_{+}=\Omega_{-}, Q_{+}=Q_{-}$and $r_{ \pm}=0$ are fulfilled and the resonant TVB oscillating with the SDFW (case i) is partitioned into two scalar single-component SIT-breathers which propagate independently of each other. Note, that in the theory of SIT we can neglect the second-order derivatives because they do not take part in the process of formation of SIT-pulses and take part only in the process of formation of the bound state of two breathers.

For the nonresonant case ii, the situation is different. The second-order derivatives in Eq. (7) not only take part in the formation of the bound state of two breathers, but also take part in the process of formation of nonresonant nonlinear solitary wave. This is explained by the fact that for the formation of nonresonant nonlinear solitary wave it is necessary that the Kerr-nonlinearity be balanced by dispersion (the diffraction effects are not considered here). Dispersion effects are connected with the term $H_{l, n}$ in Eq.(10) and if we neglect the second-order derivative terms in Eq.(77) with the coefficients $g_{2}, g_{4}, g_{5}$ then we observe that the quantity $H_{l, n}$ becomes equal to zero and the nonresonant solitary wave is not formed. So, when we investigate nonresonant nonlinear solitary waves, in contrast to SIT theory (case i) we should always take into consideration the second-order derivatives in wave equation (7).

For the hybrid TVB oscillating with the SDFW (case iii) the situation is similar to the nonresonant case because for hybrid waves both resonant and nonresonant mechanisms are involved in the formation of nonlinear solitary waves and, consequently, we must retain the second-order derivatives in the wave equation (77).

We also have to note yet another important circumstance concerning the second-order derivatives in the wave equation (7). Although we can keep the second-order derivatives in Eq.(17), nevertheless we can not always obtain TVB oscillating with SDFW. This happens so because for the investigation of two-component solitary waves it is necessary to have a sufficient number of auxiliary complex functions and parameters. In particular, the standard PRM [57], contains only one complex auxiliary function and two constant parameters which is not enough for the description of two-component solitary waves (see, for instance [53, 64, 65] ). However the application of the generalized PRM Eq.(8) gives us a chance to introduce two complex auxiliary functions and eight constant parameters.

To summarize the results discussed above, we make the following conclusions: for the investigation of resonant, nonresonant and hybrid TVB oscillating with SDFW it is necessary to take into consideration the second-order derivative terms of the wave equation (77), the waves must be the phase-modulated (see Section III), and the generalized PRM Eq.(8) should be used.

The hybrid TVB oscillating with SDFW Eq.(15) adds to new physical conditions in which allow us to study this two-component solitary wave previously had been studied only for the resonance and nonresonanse waves. On the other hand, together with hybrid one-component solitons and hybrid one-component breathers, the hybrid TVB oscillating with SDFW makes the theory of hybrid nonlinear solitary waves more complete.

\section{APPENDIX I}

$$
D_{l}=\int \varepsilon\left(z_{1}, t_{1}\right) E\left(z-z_{1}, t-t_{1}\right) d z_{1} d t_{1},
$$

Substituting Eq.(4) into Eq.(16), and taking Eq.(15) into consideration, we obtain

$$
D_{l}=\sum_{l} Z_{l}\left[\kappa-i a \frac{\partial}{\partial z}+i b \frac{\partial}{\partial t}-c \frac{\partial^{2}}{\partial z^{2}}-d \frac{\partial^{2}}{\partial t^{2}}+\tilde{t} \frac{\partial^{2}}{\partial z \partial t}\right] \hat{E}_{l},
$$

where

$$
\kappa=\int \varepsilon\left(z_{1}, t_{1}\right) e^{-i l\left(k z_{1}-\omega t_{1}\right)} d z_{1} d t_{1}
$$




$$
\begin{gathered}
a=-i \int \varepsilon\left(z_{1}, t_{1}\right) z_{1} e^{-i l\left(k z_{1}-\omega t_{1}\right)} d z_{1} d t_{1}, \\
b=i \int \varepsilon\left(z_{1}, t_{1}\right) t_{1} e^{-i l\left(k z_{1}-\omega t_{1}\right)} d z_{1} d t_{1}, \\
c=-\int \varepsilon\left(z_{1}, t_{1}\right) \frac{z_{1}^{2}}{2} e^{-i l\left(k z_{1}-\omega t_{1}\right)} d z_{1} d t_{1}, \\
d=-\int \varepsilon\left(z_{1}, t_{1}\right) \frac{t_{1}^{2}}{2} e^{-i l\left(k z_{1}-\omega t_{1}\right)} d z_{1} d t_{1}, \\
\tilde{t}=\int \varepsilon\left(z_{1}, t_{1}\right) t_{1} z_{1} e^{-i l\left(k z_{1}-\omega t_{1}\right)} d z_{1} d t_{1} .
\end{gathered}
$$

Substituting Eqs. (4) into the Bloch equations (3), and the using the condition of the inhomoheneouse broadening of the spectral line, we obtain a polarization of two-level atoms in the form

$$
P_{r}=i \frac{n_{0} \mu^{2}}{\hbar}<g>\sum_{l= \pm 1} l Z_{l}\left[\varepsilon^{1} \Theta_{l}{ }^{(1)}+\varepsilon^{2} \Theta_{l}^{(2)}+\varepsilon^{3} \Theta_{l}{ }^{(3)}-\varepsilon^{3} \frac{\kappa_{0}^{2}}{2} \int \frac{\partial \Theta_{l}^{(1)}}{\partial t} \Theta_{-l}^{(1)} \Theta_{l}^{(1)} d t^{\prime}\right]+\mathcal{O}\left(\epsilon^{4}\right)
$$

where

$$
\Theta_{l}^{(\alpha)}(z, t)=\int_{-\infty}^{t} \hat{E}_{l}^{(\alpha)}\left(z, t^{\prime}\right) d t^{\prime}, \quad<g>=\int \frac{g(\Delta) d \Delta}{1+T^{2} \Delta^{2}}
$$

$g(\Delta)$ is the inhomogeneous broadening lineshape function for an ensemble of two-level optical atoms or SQDs, $\Delta=$ $\omega_{0}-\omega$.

\section{APPENDIX II}

The analytical solution of Eq.(12) in the form of two components of the vector soliton $u_{+}$and $u_{-}$has the form (see, for instance [46, 48] and references therein)

$$
u_{ \pm}(z, t)=\frac{f_{ \pm 1}}{\mathfrak{b} T} \operatorname{sech}\left(\frac{t-\frac{z}{V_{0}}}{T}\right) e^{i\left(k_{ \pm 1} z-\omega_{ \pm 1} t\right)}
$$

where the quantities $f_{ \pm 1}, k_{ \pm 1}$ and $\omega_{ \pm 1}$ are the real constants. $V_{0}$ is the nonlinear wave velocity.

$$
T^{-2}=V_{0}^{2} \frac{v_{+} k_{+1}+k_{+1}^{2} p_{+}-\omega_{+1}}{p_{+}}, \quad \mathfrak{b}^{2}=V_{0}^{2} \frac{f_{+1}^{2} \mathfrak{q}_{+}+f_{-1}^{2} r_{+}}{2 p_{+}}, \quad k_{ \pm 1}=\frac{V_{0}-v_{ \pm}}{2 p_{ \pm}} .
$$

The following inequalities

$$
k_{ \pm 1}<<Q_{ \pm}, \quad \omega_{ \pm 1}<<\Omega_{ \pm}
$$

are valid.

The relations between the quantities $f_{ \pm 1}$ and $\omega_{ \pm 1}$ have the form

$$
f_{+1}^{2}=\frac{p_{+} \mathfrak{q}_{-}-p_{-} r_{+}}{p_{-} \mathfrak{q}_{+}-p_{+} r_{-}} f_{-1}^{2}, \quad \omega_{+1}=\frac{p_{+}}{p_{-}} \omega_{-1}+\frac{V_{0}^{2}\left(p_{-}^{2}-p_{+}^{2}\right)+v_{-}^{2} p_{+}^{2}-v_{+}^{2} p_{-}^{2}}{4 p_{+} p_{-}^{2}} .
$$

[1] G. L. Lamb, Jr., Elements of Soliton Theory (Wiley, New York, 1980). 
[2] E. G. Sauter, Nonlinear Optics (Wiley, New york, 1996).

[3] A. I. Maimistov and A. M. Basharov, Nonlinear optical waves, Kluwer Academic Publishers Dordrecht Boston, London, 643 (1999).

[4] A. P. Suchorukov, Nelineinie Volnovie Vzaimodeistvia v Optike i Padiophizike. (Nauka, Moscow, 1988).

[5] M. B. Vinogradova, O. V. Rudenko, and A. P. Suhorukov, (Theoria Voln. Nauka, Moscow, 1990).

[6] N. Akhmediev and A. Ankiewicz, Solitons, Nonlinear pulses and beams, (Chapman and Hall, London, UK, 1997).

[7] Y. S. Kivshar and G. P. Agrawal, Optical solitons. From Fibers to Photonic Crystals (Academic Press, 2003).

[8] G. T. Adamashvili and and A. A. Maradudin, Phys. Rev. E, 55, 7712-7719 (1997).

[9] S. L. McCall and E. L. Hahn, Phys. Rev. 183, 457 (1969).

[10] A. I. Maimistov, A. M. Basharov, S. O. Elyutin, and Y. M. Sklyarov, Phys. Rep. 191, 1 (1990).

[11] L. Allen and J. Eberly, Optical resonance and two level atoms (Dover, 1975).

[12] R. K. Dodd, J. C. Eilbeck, J. D. Gibbon and H. C. Morris, Solitons and Nonlinear wave Equations, Academic Press. Inc. (1982).

[13] J. C. Diels and E. L. Hahn, Phys. Rev. A 10, 2501 (1974).

[14] I. A. Poluektov, Y. M. Popov, and V. S. Roitberg, Usp. Fiz. Nauk. 114, 97 (1974).

[15] G. L. Lamb, Jr., Rev. Mod. Phys. 43, 99 (1971).

[16] M. D. Crisp, Phys. Rev. A. 2, 2172 (1970).

[17] J. E. Rothenberg, D. Grischkowsky and A. C. Balant, Phys. Rev. Lett. 53, 552 (1984).

[18] J. D. Harvey, J. M. Dudley, P. F. Curley, C. Spielmann and F. Krausz, Optics Lett. 19, 972 (1994).

[19] H. Giessen, A. Knorr, S. Haas, S. W. Koch, S. Linden, J. Kuhl, M. Hetterich, M. Grün, and C. Klingshirn, Phys. Rev. Lett. 81, 4260 (1998).

[20] G. Panzarini, U. Hohenester, and E. Molinari, Phys. Rev. B 65, 165322 (2002).

[21] S. Schneider, P. Borri, W. Langbein, U. Woggon, J. Förstner, A. Knorr, R. L. Sellin, D. Ouyang, and D. Bimberg, Appl. Phys. Lett. 83, 3668 (2003).

[22] R. M. Arkhipov, M. V. Arkhipov, I. Babushkin and N. N. Rosanov, Optics Lett. 41, 737 (2016).

[23] M. V. Arkhipov, A. A. Shimko, R. M. Arkhipov, I. Babushkin, A. A. Kalinichev, A. Demircan, U. Morgner and N. N. Rosanov , Laser Phys. Lett. 15, 075003 (2018).

[24] G. T. Adamashvili and A. Knorr, Phys. Lett. A, 367, 220 (2007).

[25] G. T. Adamashvili, C. Weber, A. Knorr, and N. T. Adamashvili, Phys. Rev. A, 75, 063808 (2007).

[26] A. A. Zabolotskii, JETP Lett.. 77, 464 (2003); JETP. 156, 890 (2019).

[27] G. T. Adamashvili and A. Knorr, Optics Letters , 31, 74 (2006).

[28] S. V. Sazonov and N. V. Ustinov, Physica D, 366, 1 (2018).

[29] G. T. Adamashvili, D. J. Kaup, A. Knorr, and C. Weber, Phys. Phys. A. 78, 013840 (2008).

[30] S. V. Sazonov and N. V. Ustinov, Physica Scripta, 94, 115208 (2019).

[31] G. T. Adamashvili, C. Weber and A. Knorr, The Eur. Phys. J. D 47, 113 (2008).

[32] G. T. Adamashvili, D. J. Kaup, and A. Knorr, Phys. Rev. A, 90, 053835 (2014).

[33] N. V. Ustinov, Preprint, Arxiv: 07052833 v1, (19 May 2007).

[34] H. Steudel, A. A. Zabolotskii, and R. Meinel, Phys. Rev. E. 72, 056608 (2005).

[35] N. N. Rozanov, V. V. Kozlov and S.Wabnitz, Phys. Rev. A 81, 043815 2010).

[36] N. V. Visotina, N. N. Rozanov and V. E. Semenov, Pisma v JTEP, 83, 337 (2006).

[37] N. V. Visotina, N. N. Rozanov, V. E. Semenov, S. V. Fedorov,and S. Wabnitz Optics and spectroscopy, 101, 736 (2006).

[38] N. Akhmediev and A. Ankiewicz, Dissipative Solitons, Lecture Notes in Physics,661, (Springer, Berlin, Heidelberg, 2005).

[39] G. T. Adamashvili, N. T. Adamashvili, M. D. Peikrishvili, G. N. Motsonelidze and R. R. Koplatadze, Technical Physics Letters, 35, 17 (2009).

[40] G. T. Adamashvili, N. T. Adamashvili, M. D. Peikrishvili, G. N. Motsonelidze and R. R. Koplatadze, Optics and spectroscopy, 106, 863 (2009).

[41] M. V. Arkhipov, A. A. Shimko, N. N. Rosanov, I. Babushkin,and R. M. Arkhipov, Phys. Phys. A. 101, 013803 (2020).

[42] M. V. Arkhipov, R. M. Arkhipov, A. A. Shimko, I. Babushkin, and N. N. Rosanov, Journal of Physics: Conference Series, 1410(1), $012102(2019)$.

[43] M. V. Arkhipov, R. M. Arkhipov, A. A. Shimko, I. Babushkin, and N. N. Rosanov, JETP Letters, 109 (10), 634 (2019).

[44] Z. Ivic, N. Lazarides, and G. P. Tsironis, Chaos, Solitons and Fractals:X,1, 100003 (2019).

[45] G. T. Adamashvili, Results in Physics, 1, 26 (2011).

[46] G. T. Adamashvili, Optics and spectroscopy, 113, 1 (2012).

[47] G. T. Adamashvili, Physica B. 407, 3413 (2012).

[48] G. T. Adamashvili, The Eur. Phys. J. D. 66, 101 (2012).

[49] G. T. Adamashvili, M. D. Peikrishvili, R. R. Koplatadze, K. L. Schengelia, Preprint, Arxiv: 1408.4310v1, (19 Aug 2014).

[50] G. T. Adamashvili, Phys. Lett. A 379, 218 (2015).

[51] G. T. Adamashvili, Optics and spectroscopy, 127, 865 (2019).

[52] G. T. Adamashvili, Preprint, Arxiv: 1907.10883v1, (25 Jul 2019).

[53] G. T. Adamashvili, The Eur. Phys. J. D, 74, Issue 3, 41 (2020).

[54] G. T. Adamashvili, Phys. Rev. E, 85, 067601 (2012).

[55] G. T. Adamashvili, Phys. Rev. E 93, 023002 (2016).

[56] G. T. Adamashvili, Preprint, Arxiv: 2001.07758v1, (21 Jan 2020). 
[57] T. Taniuti and N. Iajima, J. Math. Phys. 14, 1389 (1973).

[58] G. T. Adamashvili, N. T. Adamashvili, M. D. Peikrishvili and R. R. Koplatadze, Preprint, Arxiv:2218843, (5 Apr 2018).

[59] A. I. Maimistov and E. A. Manykin, Zh.Eksp.Teor.Fiz. 85, 1177 (1983).

[60] M. Nakazawa, E. Yamada, and H. Kubota, Phys.Rev. Lett. 66, 2625 (1991).

[61] M. Nakazawa, E. Yamada, and H. Kubota, Phys.Rev. A. 44, 5973 (1991).

[62] G. T. Adamashvili, Phys. Lett. A 310, 473 (2003).

[63] D. P. Caetano, S. B. Cavalcanti, J. M. Hickmann, A. M. Kamchatnov, R. A. Kraenkel, and E. A. Makarova, Phys.Rev.E 67, 946615 (2003).

[64] G. T. Adamashvili, Phys. Rev. E 69, 026608 (2004).

[65] G. T. Adamashvili and D. J. Kaup, Phys. Rev. E, 70, 066616 (2004).

[66] E. J. S. Fonseca, S. B. Cavalcanti,and J. M. Hickmann, Phys.Rev.E 64, 016619 (2001).

[67] G. T. Adamashvili and D. J. Kaup, Phys. Rev. E, 73, 066613 (2006).

[68] G. T. Adamashvili and D. J. Kaup, Phys. Phys. A. 99, 013832 (2019).

69] L. D. Landau and E. M. Lifshitz Electrodynamics of continuous Media (Pergamon press Ltd. New York, 1984).

[70] L. D. Landau and E. M. Lifshitz Quantum Mechanics, Nonrelativictic theory. (Pergamon press Ltd. New York, 1980).

[71] G. T. Adamashvili, M. D. Peikrishvili and R. R. Koplatadze, Technical Physics Letters, 43, 331 (2017).

[72] G. T. Adamashvili, Preprint, Arxiv: 1608.05349v1, (18 Aug 2016).

[73] G. T. Adamashvili, Acoustical Physics, 63, 517 (2017). 